\title{
Médiévales
}

Langues, Textes, Histoire

69 | automne 2015

Travailler à Paris (XIIIe-XVIe siècle)

\section{L'alimentation, un risque pour la santé ? Discours médical et pratiques alimentaires au Moyen Âge}

Food, a Risk for Health? Medical Discourse and Dietary Practices in the Middle Ages

\section{Marilyn Nicoud}

\section{OpenEdition}

Journals

Édition électronique

URL : https://journals.openedition.org/medievales/7634

DOI : $10.4000 /$ medievales.7634

ISSN : 1777-5892

Éditeur

Presses universitaires de Vincennes

Édition imprimée

Date de publication : 30 novembre 2015

Pagination : 149-170

ISBN : 978-2-84292-444-7

ISSN : 0751-2708

\section{Référence électronique}

Marilyn Nicoud, «L'alimentation, un risque pour la santé ? Discours médical et pratiques alimentaires au Moyen Âge », Médiévales [En ligne], 69 | automne 2015, mis en ligne le 30 novembre 2017, consulté le 22 avril 2022. URL : http://journals.openedition.org/medievales/7634 ; DOI : https://doi.org/

10.4000/medievales.7634 
Marilyn Nicoud

\section{L'alimentation, un risque pour la santé ? Discours médical et pratiques alimentaires au Moyen Âge}

Il peut paraître à première vue surprenant de chercher à définir le rôle qu'a pu jouer le savoir des médecins dans la manière de penser les dangers, voire les risques alimentaires, dans les sociétés anciennes, quand on constate les difficultés qu'ils ont, aujourd'hui encore, à imposer leur statut d'expert lorsque se posent des questions de sécurité alimentaire ${ }^{1}$. Si l'on a certes recours à leurs compétences, en particulier dans le domaine de la qualité sanitaire des aliments (Food Safety) ${ }^{2}$, les conflits d'intérêts, mais aussi la difficulté à obtenir des résultats scientifiques certains fragilisent souvent leur position ${ }^{3}$. Au mieux, les pouvoirs publics choisissent alors d'imposer des mesures restrictives à l'égard de certains produits, en vertu généralement d'un principe de précaution.

Pour des périodes plus éloignées et notamment pour le Moyen Âge, il s'avère difficile de trouver le témoignage direct d'expertises médicales en matière alimentaire à l'échelle des collectivités ${ }^{4}$. Cela ne signifie pas

1. Je remercie les relecteurs de cet article, D. Boisseuil, B. Laurioux et L. Moulinier, pour leurs conseils.

2. La qualité sanitaire des produits recouvre, avec le domaine de la sécurité des approvisionnements (Food Security), la notion de sécurité alimentaire.

3. $C f$. M. Bruegel, A. Stanziani, "Pour une histoire de la sécurité alimentaire », Revue d'histoire moderne et contemporaine, 51/3 (2004), La Sécurité alimentaire, entre santé et marché, p. 7-16.

4. Il est possible que les connaissances médicales sur les pathologies animales ou sur les effets induits par une alimentation malsaine sur la santé humaine aient influencé dans une certaine mesure les décisions prises par les autorités publiques, mais on ne dispose pas de sources pour l'étayer : voir M. Ferrières, Histoire des peurs alimentaires. Du Moyen Âge à l'aube $d u X X^{e}$ siècle, Paris, 2002, p. 17-42, qui met en relation les décisions politiques et le discours médical. Mais en règle générale, l'expertise en matière alimentaire reste du ressort des métiers. Sur cette notion d'expertise, voir B. Laurioux, « L'expertise en matière d'alimentation au Moyen Âge. Problèmes, méthodes et perspectives », dans C. Denjean, 
que les autorités n'ont pas, de leur côté, apprécié et tenté d'évaluer, dans une certaine mesure, les risques que l'absence ou la corruption de certaines denrées primordiales pouvaient faire courir à la santé publique ${ }^{5}$ : entendus aussi bien d'un point de vue quantitatif que qualitatif, ces risques relèvent dans un cas de la pénurie ou de la disette, les plus redoutées car les plus radicales par leurs conséquences, et dans l'autre de la catégorie du malsain ou du nocif dont les effets, généralement non épidémiologiques, ne sont pas moins importants ${ }^{6}$. Délibérations citadines, statuts communaux et de métiers, archives de magistratures spécialisées et judiciaires offrent autant de témoignages, dès le Moyen Âge, de ces efforts d'intervention des pouvoirs publics pour tenter de réduire, autant que possible, de tels risques ${ }^{7}$. Mais nulle trace, en général, dans ces sources d'une participation des praticiens, dont certains sont pourtant employés par les autorités urbaines,

L. Feller éd., Expertise et valeur des choses au Moyen Âge, vol. 1 : Le besoin d'expertise, Madrid, 2013 (Collection de la Casa de Velázquez, 139), p. 19-35.

5. Sur les sources archivistiques en matière de ravitaillement, de contrôle de l'approvisionnement et des prix dans l'espace italien médiéval et moderne, voir « Le magistrature e le istituzioni alimentari », dans Gli archivi per la storia dell'alimentazione. Atti del convegno (Potenza-Matera, 5-8 settembre 1988), vol. 1, Rome, 1995 (Pubblicazioni degli Archivi di Stato, 34), p. 285-714.

6. Sur la question des disettes et des famines, la bibliographie est trop importante pour en faire ici état. Je me contente de renvoyer à des références récentes : M. Bourin, J. Dendrel, F. Menant éd., Les Disettes dans la conjoncture de 1300 en Méditerranée occidentale, Rome, 2011 (CEFR, 450) ; P. Benitoi Monclús, Crisis alimentarias en la Edad Media, Lérida, 2012. Cette question a été au cœur aussi des interrogations des historiens de l'École des Annales lorsqu'ils se sont intéressés à l'histoire de l'alimentation en des termes plutôt quantitatifs (rations, consommations, conjoncture). Pour une mise au point historiographique, voir O. Redon, B. Laurioux, " Histoire de l'alimentation entre Moyen Âge et Temps modernes. Regards sur trente ans de recherches », dans O. Redon, L. Sallmann, S. Steinberg éd., Le Désir et le Goût. Une autre histoire (XIII ${ }^{e}$-XVIII ${ }^{e}$ siècles), Saint-Denis, 2005, p. 54-96. Sur les risques encourus du fait de la consommation d'aliments nocifs, voir M. Ferrières, Histoire des peurs alimentaires..., qui souligne notamment l'importance de la réglementation communale dans les espaces méditerranéens en matière de boucherie et la confronte aux législations des métiers en vigueur dans la France d'oïl. Les ventes de poissons et de pain sont aussi généralement régulées par les instances publiques. Pour les métiers de la boucherie, voir B. Descamps, " "Sera veu et extimé par les jurez et gens en ce cognoissans". Procédures de contrôle dans la boucherie parisienne à la fin du Moyen Âge », dans Expertise et valeur des choses..., p. 53-63.

7. Voir par exemple A. M. Nada Patrone, «Gli statuti comunali come fonte per la storia dell'alimentazione », dans Gli archivi per la storia dell'alimentazione..., vol. 1, p. 637-647 ; L. Castellazzi, « Legislazione sanitaria per l'alimentazione a Verona in epoca veneta ", dans ibid., vol. 2, p. 879-893. Pour une étude des habitudes alimentaires à partir des archives communales, voir A. M. Nada Patrone, Il cibo del ricco ed il cibo del povero. Contributo alla storia qualitativa dell'alimentazione. L'area pedemontana negli ultimi secoli del Medio Evo, Turin, 1989 (Biblioteca di « Studi Piemontesi »). 
dans les espaces méditerranéens notamment, afin d'assurer un minimum de protection sanitaire aux populations ${ }^{8}$.

Si l'on cherche les prémisses d'une expertise médicale dans le domaine alimentaire, c'est à une autre échelle qu'il faut se situer, celle de la santé individuelle ${ }^{9}$. Loin d'être rares, ces expertises, assez diffusées, se fondent sur une conception toute particulière du rôle dévolu aux nourritures dans la santé et la pratique médicale. L'héritage en est ancien puisqu'il remonte aux premiers traités de la tradition hippocratique. Déjà, à la fin du $v^{\mathrm{e}}$ siècle av. J.-C., l'auteur d'Ancienne médecine, situant l'origine de la médecine dans l'invention de la cuisine, soulignait l'aptitude progressive des hommes à distinguer les nourritures conformes à leurs capacités naturelles, à les apprêter convenablement, à écarter les substances « à l'origine [de] souffrances, maladies et mort » et à choisir les aliments synonymes de « nourriture, accroissement et santé ${ }^{10}$. Surtout, considérant l'alimentation comme un remède mais aussi comme une cause d'affection, c'est-à-dire comme une substance potentiellement à risque pour la santé des individus $\mathrm{du}$ fait de son ambivalence, les praticiens antiques puis médiévaux ont suscité la naissance d'une branche de la médecine appelée diététique (ars diaetae), pour partie consacrée à la connaissance et à l'usage des nourritures à des fins thérapeutiques, préventives et conservatoires ${ }^{11}$.

L'alimentation constitue donc un élément fondamental dans les conceptions antiques et médiévales de l'art médical : son application seule (au sein d'un régime de vie), ou accompagnée d'une pharmacopée adaptée, voire d'une intervention chirurgicale, peut permettre à un patient de se rétablir. En matière de conservation de la santé, le médecin souligne la nécessité de se nourrir convenablement, de connaître les produits les plus adaptés à sa propre complexion et de savoir distinguer les aliments propres

8. Sur la genèse du praticien communal, voir V. Nutton, « Continuity or Rediscovery ? The City Physician in Classical Antiquity and Medieval Italy », dans A. W. Russel éd., The Town and State Physician in Europe, from the Middle Ages to the Enlightenment, Wolfenbüttel, 1981, p. 9-46, rééd. dans V. Nutton, From Democedes to Harvey, Aldershot, 1988. Pour une étude de cas, voir M. R. McVaugh, "Le coût de la pratique et l'accès aux soins. L'exemple de la ville catalane de Manresa », Médiévales, 46 (2004), Éthique et pratique médicale, p. 45-54.

9. Pour une première approche sur l'utilité d'une réflexion diachronique en matière de nutrition et sur l'apport des médecins médiévaux dans ce domaine, voir M. Pouillart, P. Pouillart, «L'expertise culinaire du médecin au Moyen Âge au centre des pratiques éducatives alimentaires en France », Cahiers de nutrition et de diététique, 46 (2011), p. 247258.

10. Hippocrate, Ancienne médecine, texte établi et traduit par J. Jouanna, Paris, 1990 (CUF), III, 5. Sur ces questions, voir D. Jacquart, « La nourriture et le corps au Moyen Âge », Cahiers de recherches médiévales (XII ${ }^{e}$-XV $V^{e}$ siècles), 13 (2006), p. 259-266.

11. L'ars diaetae est, dans les conceptions antique et médiévale, synonyme d'hygiène : voir F. Audoin-Rouzeau, F. Sabban éd., Un aliment sain dans un corps sain. Perspectives historiques, Tours, 2007. 
à l'homme sain de ceux qui sont destinés au malade, le régime de l'un et de l'autre n'étant pas identiques.

En un sens, le risque alimentaire (au sens d'une ambivalence du rôle et des effets reconnus de l'aliment - remède ou danger), plus que les risques alimentaires, fait donc partie à part entière des conceptions médicales médiévales - mais aussi actuelles — , puisque se nourrir peut aussi bien permettre à l'individu de conserver sa santé que de la perdre ${ }^{12}$. Ce risque, quotidien et individuel, n'est pas le même pour tous et malgré les mises en garde prodiguées par les praticiens, il n'est peut-être pas toujours perceptible, voire facilement concevable par les consommateurs. Aussi des décalages peuvent-ils parfois se vérifier entre le plaisir que certaines nourritures procurent et le risque qu'elles représentent, ce qui pose le problème de la réception et de la diffusion du savoir médical hors de la sphère professionnelle.

\section{Les médecins médiévaux et l'alimentation}

Élément indispensable à la vie, mais aussi danger pour la santé, l'aliment est un sujet abondamment traité dans la littérature médicale, qu'elle concerne les aspects théoriques, destinés plutôt à des praticiens, ou les aspects pratiques à l'usage d'un plus vaste public.

\section{Aliments et nutrition dans les conceptions médicales}

Les conceptions médicales du Moyen Âge, héritières de leurs devancières grecques et de langue arabe, attribuent un rôle spécifique à l'alimentation qui dépasse celui qui est dévolu à l'ensemble des « choses non naturelles ${ }^{13}$; non seulement, comme ces dernières, elle permet de conserver l'équilibre des complexions qui définit l'état de santé mais, en plus, elle sert à restaurer le corps. «Elle reconstitue au sens propre du terme les substances dont il est formé, au fur et à mesure de la consomption de celles-ci sous l'effet de activités qu'accomplit le corps grâce à des facultés ou "vertus"14. » Cela signifie que l'aliment ingéré est destiné à devenir

12. $C f$. M. Ferrière, « La perception du risque alimentaire : une histoire de longue durée », dans L'Alimentation à découvert, sous presse.

13. Ce terme désigne, dans le vocabulaire médical médiéval, un ensemble de paramètres (que Galien appelait les « choses nécessaires ») liés à l'environnement et indispensables à la vie. Au nombre de six - air, alimentation et boisson, sommeil et veille, inanition et réplétion, exercice et repos, passions de l'âme, auxquels sont souvent associés le bain et l'activité sexuelle -, ces choses sont nécessaires au maintien de la santé, mais mal utilisées, elles sont sources possibles de dérèglement. Elles constituent aussi des instruments thérapeutiques aux côtés de la pharmacopée notamment.

14. D. Jacquart (« La nourriture et le corps... », p. 260) décrit et analyse précisément l'apport de la nourriture au corps selon les conceptions médicales, antiques et médiévales. 
matière corporelle ; il est transformé lors du processus complexe de digestion et il donne naissance aux humeurs qui façonnent à leur tour les os, les veines, les nerfs, mais aussi les organes et les membres, bref tout ce qui constitue la charpente extérieure et l'organisation interne du corps humain. Tout au long des différentes coctions qui s'effectuent successivement dans l'estomac, dans le foie, dans les veines, le produit des opérations, lorsqu'il est utile, est conservé, et rejeté lorsqu'il ne l'est point.

Quoique, dans une certaine mesure, la transformation de l'aliment en substance corporelle permette, au gré des cuissons, de séparer « le bon grain de l'ivraie », tout aliment n'est pas bon à consommer, ou plus exactement n'est pas adapté à n'importe quel consommateur. Manger sans connaître au préalable ce que l'on ingère revient à courir un risque au sens de l'une des étymologies de ce $\operatorname{mot}^{15}$ : ici il s'agit de s'exposer à la bonne ou à la mauvaise fortune ${ }^{16}$. La «probabilité de dommage ${ }^{17}$ »qui en découle est toutefois difficile à évaluer. Théoriquement, s'il est bien choisi, l'aliment s'adapte parfaitement à la nature du consommateur et doit avoir des effets bénéfiques ; dans le cas contraire, il est facteur de maladie. Toutefois, les médecins reconnaissent aussi qu'un aliment a priori nocif pour un individu pourra se révéler acceptable si, par exemple, on le consomme depuis longtemps avec appétit ${ }^{18}$.

Au-delà de ces cas particuliers, les conceptions médicales en matière d'aliments et d'alimentation participent d'un schéma d'explication général. À l'image de l'être humain et plus largement de toutes les composantes du monde sublunaire, l'aliment a été constitué au moment de la Création, à

Voir aussi J. Cadden, «Albertus Magnus' Universal Physiology : the Example of Nutrition », dans J. A. Weisheipl éd., Albertus Magnus and the Sciences. Commemorative Essays, Toronto, 1980, p. 321-341.

15. Notons d'emblée toutefois que le mot « risque » ou, plus exactement, son équivalent latin n'est pas employé par les médecins dans leurs écrits.

16. Au sens de l'arabe «rizq », terme qui serait peut-être à l'origine du mot risque : $c f$. M. de Epalza, "Origines du concept de risque : de l'Islam à l'Occident », dans L. Faugères, P. Vasarhelyi, C. Villain-Gandossi éd., Le Risque et la Crise, Malte, 1990, p. 63-70. Voir aussi, sur la polysémie de l'emploi de risque en latin (resecum) et dans les langues vulgaires, B. Marin, « Dire le risque. Risque, riescho, riesgo et leurs usages », dans G. Chastagnaret éd., Les Sociétés méditerranéennes face au risque. Discipline, temps, espace, Le Caire, 2008, p. 13-41.

17. C. Villain-Gandossi, "Une analyse de l'approche globale des risques », dans P. Gabert et J. Vaudour éd., Les Risques naturels, $120^{\circ}$ congrès des sociétés historiques et scientifiques (Aix-en-Provence, 1995), Paris, 1999, p. 11-21 (p. 18).

18. C'est ce que déclare le médecin Benedetto Reguardati (1398-1469) à l'évêque Astorgius Agnese, le destinataire de son régime, à propos du fromage qu'il déconseille de manière générale, tout en reconnaissant que l'appétit que son patient éprouve pour cet aliment et sa longue habitude de consommation sont des facteurs qui en atténuent la nocivité ( « Nec vos conturbet pater sancte quod caseus ita vobis familiaris existat cibus, quia propter longam consuetudinem et aviditatem appetitus ad illum in vobis maxima pars suorum nocumentorum aufertur », Città del Vaticano, Biblioteca Apostolica Vaticana, Vat. lat. 6266, $\mathrm{f}^{\circ} 31 \mathrm{r}^{\circ}$ ). 
partir des quatre éléments qui ont été transformés en humeurs ${ }^{19}$. Chacune d'entre elles est caractérisée par une complexion, c'est-à-dire par un mélange de qualités premières plus ou moins harmonieux ${ }^{20}$. Du fait du principe d'attirance du semblable par le semblable, et en raison de la perception de la santé en terme d'équilibre (et de la maladie comme un déséquilibre), il convient à l'homme sain de choisir des aliments identiques à sa complexion, facilement assimilés par l'organisme et, au malade, de se nourrir d'aliments contraires, susceptibles de l'aider à retrouver sa santé perdue.

\section{Production textuelle}

Ces principes font manifestement partie d'un bagage général de connaissances diététiques assez bien diffusé et dans une large mesure partagé. Mais les recommandations des médecins visent aussi, par une bonne connaissance respective de la nature du patient et des aliments, à réduire la part de risque que ces derniers contiennent. L'objectif poursuivi justifie sans doute l'intérêt soutenu que les praticiens antiques puis médiévaux ont accordé à ces aspects et l'importance de la production textuelle qui en découle. Partant de la tradition Du régime pseudo-hippocratique, et d'une série d'autres ouvrages de l'héritage antique et arabe, la littérature médiévale sur le sujet s'est en effet très largement développée et diversifiée.

Durant le haut Moyen Âge, quoique les soubassements théoriques du discours médical aient été assez limités, les aspects alimentaires étaient cependant déjà relativement présents dans la littérature médicale, aussi bien à travers des calendriers par mois ou par saisons qui indiquaient ce qu'il fallait consommer ${ }^{21}$, que sous la forme de listes énumérant ce qui était bon pour la santé ou plus particulièrement pour certaines parties du corps, et ce qui était nocif : ces De conferentibus et nocentibus, dont la tradition se poursuit tout au long du Moyen Âge, ne délivrent, en règle générale, aucune notice

19. $C f$. D. Jacquart, « La nourriture et le corps... ».

20. «Plus ou moins harmonieux », en effet, car il n'existe pas à proprement parler dans la nature de mélange parfait des quatre qualités ; au contraire on parle plutôt de complexion sanguine (chaude et humide), colérique (chaude et sèche), mélancolique (froide et sèche) et flegmatique (froide et humide), car même dans un état de santé, domine chez les individus l'un de ces mélanges. On parlera donc plutôt de « latitude de santé » (latitudo sanitatis). Cf. P.-G. Ottosson, Scholastic Medicine and Philosophy. A Study of Commentaries on Galen's «Tegni » (ca 1300-1450), Naples, 1984.

21. Il ne faut pas oublier non plus quelques rares exemples de textes entièrement dédiés à l'alimentation, lointaine préfiguration des régimes de santé médiévaux, tel le De observatione ciborum ad Theodoricum regem Francorum epistula d'Anthime (éd. E. Liechtenhan, 8.1, Corpus Medicorum Latinorum, Leipzig, 1928, rééd. 1963). Pour une étude de ces ouvrages, voir F. Pucci Donati, Dieta, salute, calendari. Dal regime stagionale antico ai regimina mensium medievali : origine di un genere nella letteratura medica, Spolète, 2007. 
explicative à leurs recommandations ${ }^{22}$. À partir du xiiie siècle, dans le cadre d'une médecine scolastique désormais enseignée à l'Université, sur la base d'un curriculum d'études et d'un programme de textes à commenter ${ }^{23}$, le savoir sur l'alimentation, loin de disparaître, se développe et se ramifie dans des types d'ouvrages différents : d'abord dans des traités de langue grecque ou arabe traduits en latin qui, très vite, deviennent des outils fondamentaux de l'enseignement médical. Certains d'entre eux sont très largement consacrés aux nourritures, tels le De facultatibus alimentorum de Galien, traduit par deux fois au xiii ${ }^{\mathrm{e}}$ siècle, ou encore le De diaetis universalibus et particularibus du médecin juif de Kairouan, Isaac Israëli ( $\mathrm{x}^{\mathrm{e}}$ siècle) ; cette œuvre, entièrement consacrée à l'alimentation, fut partiellement traduite dès le xi ${ }^{\text {e }}$ siècle par Constantin l'Africain ${ }^{24}$. Ces volumes figurent en bonne place dans les cursus universitaires, notamment à Paris (statuts de 1270). Le discours diététique prend aussi place dans le genre des commentaires, qui constitue l'une des méthodes d'enseignement médiéval ${ }^{25}$, lorsqu'il s'agit de gloser des traités qui, à l'image de certains passages du premier livre du Canon d'Avicenne, ou de l'ensemble des Diètes d'Isaac, se consacrent aux vertus et nocivités des produits consommés. Fréquentes y sont les questions qui traitent de la nature de l'aliment, du processus de digestion ou encore de l'ordre de consommation des mets ${ }^{26}$.

La diététique intervient aussi dans des textes de la pratique, tant dans les conseils ${ }^{27}$ (sortes de prescriptions médicales apparues dans le dernier tiers du xiii ${ }^{e}$ siècle) que dans les practicae qui envisagent l'étiologie et le

22. Une certaine tradition médiévale attribue l'un de ces De conferentibus et nocentibus à Arnaud de Villeneuve. Cf. J. A. Paniagua, «El maestro Arnau de Vilanova, médico », dans Id., Studia Arnaldiana. Trabajos en torno a la obra médica de Arnau de Vilanova, ca 12401311, Barcelone, s.d. (1995), p. 51-143 (p. 122).

23. D. Jacquart, « La scolastique médicale », dans M. D. Grmek éd., Histoire de la pensée médicale en Occident, vol. I, Antiquité et Moyen Âge, Paris, 1995, p. 175-210.

24. Sur la transmission des textes arabes en Occident, voir D. Jacquart, F. Micheau, La Médecine arabe et l'Occident médiéval, Paris, 1990.

25. J. Agrimi, C. Crisciani, "Edocere medicos ». Medicina scolastica nei secoli XIIIXV , Naples, 1988 ; D. Jacquart, « La question disputée dans les facultés de médecine », dans B. C. Bazàn, G. Fransen, J. W. Wippel, D. Jacquart, Les Questions disputées et les questions quodlibétiques dans les facultés de théologie, de droit et de médecine, Turnhout, 1985 (Typologie des sources du Moyen Âge occidental, 44-45), p. 281-315.

26. Voir par exemple la liste des questions traitées par le bolonais Taddeo Alderotti et par ses élèves dans leurs commentaires aux textes d'autorités ( $c f$. N. G. Siraisi, Taddeo Alderotti and his Pupils. Two Generations of Italian Medical Learning, Princeton, 1981, p. 313, 330333). Pour un exemple de « lectures » d'un texte universitaire consacré à l'alimentation, voir M. Nicoud, « Les marginalia dans les manuscrits latins des Diètes d'Isaac Israëli conservés à Paris », dans D. Jacquart, C. S. F. Burnett éd., "Scientia in margine ». Études sur les " marginalia » dans les manuscrits scientifiques du Moyen Âge à la Renaissance, Genève, 2005, p. 191-215.

27. Voir J. Agrimi, C. Crisciani, Les « Consilia » médicaux, Turnhout 1993 (Typologie des sources du Moyen Âge occidental, 69). 
traitement des maladies en fonction de leur localisation dans le corps. En règle générale, tout particulièrement dans les consilia, la partie thérapeutique comprend toujours, aux côtés de préparations pharmacologiques, une diète (diaeta) souvent très largement consacrée à l'alimentation ${ }^{28}$. Certaines œuvres traitent du seul régime du malade à l'image d'un opuscule, le De modo preparandi cibos et potus infirmorum, rédigé au xii ${ }^{\mathrm{e}}$ siècle par le maître salernitain Petrus Musandinus ${ }^{29}$, qui eut un grand succès si l'on en croit le nombre de manuscrits conservés. Dans un registre proche, on pourrait aussi citer les Tabule que medicum informant specialiter cum ignoratur egritudo faussement attribuées au célèbre médecin de Montpellier, Arnaud de Villeneuve ( $c a$ 1240-1311), et qui énumèrent aliments et médicaments pour les malades ${ }^{30}$, ou encore le Tractatus de cibis attribué à son collègue Bernard de Gordon $(c a \text { 1258-1318 })^{31}$. Enfin, on ne saurait passer sous silence une autre catégorie de textes qui, pour sa part, met tout particulièrement en évidence l'aspect conservatoire, voire préventif, de l'alimentation : les régimes de santé ${ }^{32}$; souvent destinés à des individus particuliers, ils attribuent, au côté des autres composantes de la diététique, une grande place aux vertus et nocivités des nourritures. Quelques médecins en sont même venus, à l'image de Barnabas Riatinis (ca 1300-ca 1365) ${ }^{33}$, originaire de Reggio d'Émilie, du florentin Bernardo Torni (1452-1497) ${ }^{34}$ et du piémontais Pantaleone da Confienza $\left(\dagger c a\right.$ 1497) ${ }^{35}$, à consacrer leur propos aux seuls aliments, voire à un seul d'entre eux.

28. Pour un exemple spécifique, voir la collection de consilia du praticien siennois Ugo Benzi, analysée par D. P. Lockwood, Ugo Benzi Medieval Philosopher and Physician 13761439, Chicago, 1951.

29. S. De Renzi, Collectio salernitana, 4, Naples, 1855 , p. 558-565, et surtout B. Laurioux, «Petrus Musandinus et son traité sur l'alimentation des malades », dans D. Jacquart, A. Paravicini Bagliani éd., La scuola medica salernitana. Gli autori e $i$ testi, Florence, 2007, p. 235-260.

30. Le traité est édité à la Renaissance dans les Opera omnia d'Arnaud de Villeneuve.

31. Sur ce médecin, voir L. Demaitre, Doctor Bernard de Gordon, Professor and Practitioner, Toronto, 1980.

32. P. Gil Sotres, "Les régimes de santé », dans Histoire de la pensée médicale..., p. 257-281; Id., «Els regimina sanitatis», dans Regimen sanitatis ad regem Aragonum, Arnaldi de Villanova Opera omnia medica, t. X.1, éd. L. García Ballester, M. R. McVaugh, Barcelone, 1996, p. 25-394 ; M. Nicoud, Les Régimes de santé au Moyen Âge. Naissance et diffusion d'une écriture médicale, 2 vol., Rome, 2007 (BEFAR, 333).

33. Le médecin émilien se limite en effet dans son Compendium de naturis et proprietatibus alimentorum rédigé en 1337 à parler des aliments. $C f$. M. Nicoud, « L'adaptation du discours diététique aux pratiques alimentaires : l'exemple de Barnabas de Reggio », Mélanges de l'École française de Rome. Moyen Âge, 107/1 (1995), p. 207-231.

34. Bernardo Torni, plus d'un siècle plus tard, est l'auteur de deux régimes uniquement centrés sur l'alimentation ; l'un se limite même aux nourritures de temps de Carême : cf. M. Messina, « Bernardo Torni », Physis, 17 (1975), p. 249-254.

35. Pour le Moyen Âge, le premier exemple est fourni par la Summa lacticiniorum de Pantaleone da Confienza écrite dans le dernier tiers du xve siècle $: c f$. Formaggi del Medioevo. 
Que nous disent ces textes ? Dans quelle mesure entretiennent-ils le lecteur, qu'il soit confrère, étudiant en médecine, ou néophyte en la matière, de la question du risque encouru en s'alimentant et quelle est la nature de ce risque?

\section{L'alimentation, une fonction ambivalente}

$\mathrm{Au}$ sein de l'abondante production médicale citée, qui traite de manière partielle ou plus approfondie de l'alimentation, on distinguera d'un côté les ouvrages de l'enseignement médical qui livrent une conception théorique du rôle et de la nature des nourritures, et qui proposent une classification des substances ingérées ; et, de l'autre, sur le versant pratique ${ }^{36}$, les régimes de santé : s'adressant en effet de manière privilégiée à des nonspécialistes afin de leur délivrer des recommandations de bonne conduite en matière d'hygiène et développant une approche plutôt conservatoire, voire préventive, du rôle de l'aliment, ces derniers sont les plus à même de définir le risque alimentaire et d'en objectiver les dangers.

\section{Essai de typologie du risque alimentaire}

La lecture de la production médicale médiévale permet de fournir une sorte de typologie du risque et de son rapport avec la question des responsabilités. Le risque encouru par l'homme peut être d'ordre qualitatif (mal manger) ou quantitatif (trop manger ou manger insuffisamment). La responsabilité en incombe soit à l'aliment lui-même, qui pour l'essentiel est la cause d'un risque plutôt qualitatif (ingestion d'une nourriture nocive, voire d'un poison, consommation d'un «aliment-médicament » et non d'un « aliment naturel ${ }^{37}$ ), soit au consommateur, responsable d'un risque aussi bien qualitatif (mauvais choix alimentaire) que quantitatif (une alimentation trop abondante, par exemple) (fig. 1). Ce schéma général, déduit de la conception médicale, rejoint la définition qui est donnée du risque, causé soit par l'état d'un phénomène ou d'une situation (qui serait ici la nature propre de l'aliment), soit par le comportement humain (ici le

La «Summa lacticiniorum » di Pantaleone da Confienza, éd. I. Naso, Turin, 1990, rééd. dans Ead., Università e sapere medico nel Quattrocento. Pantaleone da Confienza e le sue opere, Cuneo-Verceil, 2000.

36. La médecine, à la fois science et art, est constituée en effet de deux branches, l'une théorique et l'autre pratique.

37. Je reviendrai sur ces définitions dans la sous-partie suivante consacrée à la classification des substances. 


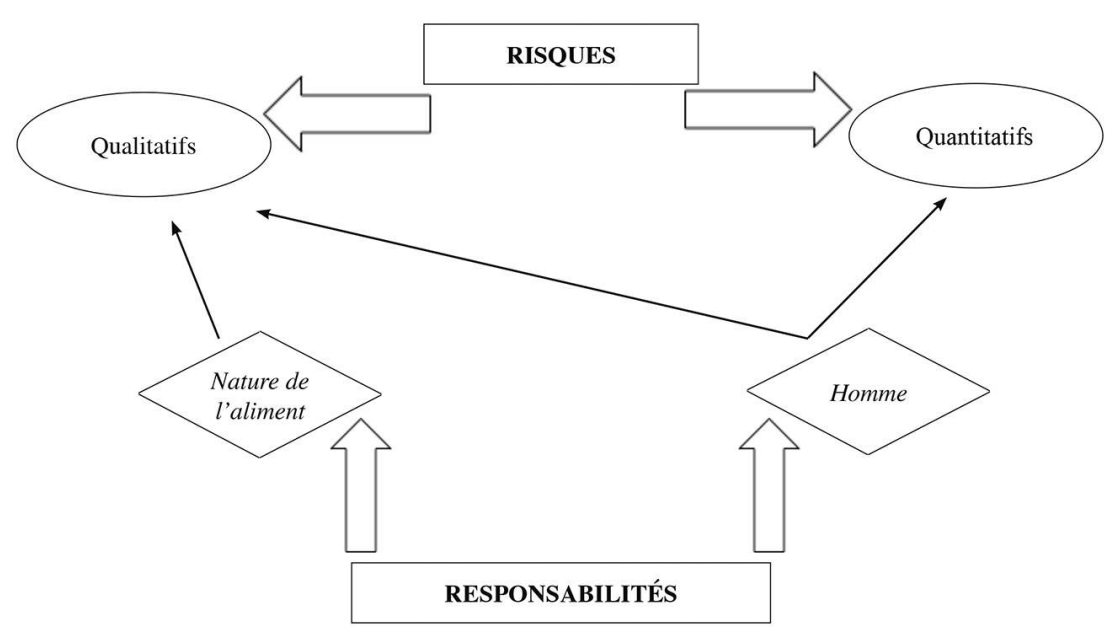

Fig. 1. La conception du risque d'après les ouvrages médicaux médiévaux

fait de se nourrir) ${ }^{38}$. En un sens s'y distinguent ce qu'on pourrait appeler, respectivement, risque naturel et risque accidentel.

Dans le cas d'un risque quantitatif, c'est moins le manque ou la disette qui sont évoqués par les médecins que le «trop manger ». L'excès de nourriture est en effet très souvent cité, au point parfois de devenir un topos des régimes de santé. Rien d'étonnant à cela, puisque ces textes s'adressent en premier lieu à des personnes qui ne connaissent point le rationnement et le manque, mais bien plutôt la profusion alimentaire. Aussi les dénonciations de la gloutonnerie ( $g u l a)$, responsable de nombre de maladies, sont-elles légion : d'abord, sans doute, car il s'agit pour les auteurs de régimes de critiquer des attitudes très répandues dans les sociétés de cours qu'ils fréquentent, le luxe et l'ostentation alimentaire faisant non seulement partie des plaisirs personnels mais, en quelque sorte aussi, d'une éthique de comportement conforme à ces milieux ${ }^{39}$. Les usages curiaux, qui supposent de manger plus que nécessaire et de goûter à une grande diversité de plats au cours d'un même repas, provoquent des troubles digestifs. La critique de telles habitudes s'accompagne bien souvent aussi, dans les écrits des médecins médiévaux, d'un discours aux tonalités moralisantes lorsqu'il s'agit d'opposer la gula des Modernes et l'idéal de sobriété, voire de frugalité des Anciens, en partie hérité du modèle de tempérance aristotélicien et de la

38. C. Villain-Gandossi, «Une analyse de l'approche... », p. 17.

39. Pour un exemple particulier des difficultés à imposer tempérance et modération, notamment en matière alimentaire, voir M. Nicoud, « Les médecins à la cour de Francesco Sforza ou comment gouverner le prince (deuxième moitié du $\mathrm{xv}^{\mathrm{e}}$ siècle) », dans Le Désir et le Goût..., p. 201-217. 
tradition chrétienne - puisque la gula est aussi l'un des péchés capitaux ${ }^{40}-$, mais sans doute aussi en partie inventé.

Les catégories du «mal manger » (ordre qualitatif) sont nombreuses et mettent en cause aussi bien le consommateur que le produit lui-même. Distinguer les responsabilités respectives n'est pas toujours chose aisée. S'alimenter à un mauvais moment (par rapport aux saisons de l'année, au déroulement de la journée - avant l'exercice par exemple -, ou quand on n'a pas faim), choisir des nourritures non appropriées à sa complexion ou plus simplement à certaines de ses dispositions, ou encore des plats mal préparés (mauvaise cuisson ou accompagnements inadaptés), relèvent plutôt de la responsabilité individuelle. Arnaud de Villeneuve, auteur du régime de santé le plus diffusé au Moyen Âge, énumère les conséquences de telles consommations et les précautions à prendre dans le chapitre de son traité consacré au fait de s'alimenter : il convient d'abord, lors des repas, de ne manger que lorsqu'on a faim, sous peine que «l'aliment ne se corrompe plus qu'il ne se transforme en membre ${ }^{41}$, et ne pas tolérer longtemps la faim, si ce n'est lorsque « les saintes constitutions de l'Église, l'occupation honnête ou la dévotion religieuse suggèrent parfois de [la] tolérer ${ }^{42}$. Il faut aussi bien mastiquer, sous peine de risquer de manger avec trop d'avidité et de rendre plus difficile le processus de digestion ${ }^{43}$. Enfin, « on ne doit pas

40. Je n'insisterai pas ici sur cet aspect de tempérance déjà présent dans les mises en garde de la diététique grecque (à propos notamment de la diète des athlètes). Le modèle médiéval, tel qu'il est préconisé par les régimes de santé, insiste sur un idéal de modération et de tempérance proche, à bien des égards, des recommandations qui figurent par exemple dans l'Éthique à Nicomaque. Les mises en garde contre la gula sont toutefois loin d'être propres au discours médical ; occupant dans le septénaire des péchés capitaux, une place non négligeable, la gourmandise est aussi largement présente dans les propos des théologiens. Sur ces aspects, C. Casagrande, S. Vecchio, Histoire des péchés capitaux au Moyen Âge, trad. fr., Paris, 2002.

41. «De corpore namque sano tunc est certum quod indiget cibo quando manifeste famescit ; [...] Propterea magis corrumpitur in eis quam convertatur in ea, nam cum respuant ipsum vel non conantur vel tepide in substanciam suam convertere » (Regimen sanitatis ad regem Aragonum... », p. 429).

42. "Quamobrem patet ex hiis quod sanis corporibus in qualibet parte anni nulla secundum naturam est alia congrua hora cibacionis, sive in prandio sive in cena, nisi quando natura comedere appetit. [...] Sumendus est ergo cibus appetente natura, nec diu eciam post esuriem deberet sumpcio tardari, nisi quia sancte constituciones ecclesie vel honesta occupacio aut religiosa devocio quandoque suggerunt ipsam esuriem tolerare » (ibid.). Notons que les régimes de santé ne font généralement pas référence à ces aspects religieux qui imposent au chrétien un calendrier particulier. La mention s'explique ici par les intérêts théologiques de l'auteur, dont les œuvres médicales voisinent avec une production spirituelle et eschatologique. Cf. J. Ziegler, Medicine and Religion ca 1300 : The Case of Arnau de Vilanova, Oxford, 1998.

43. "Leduntur autem ex cibo imperfecte masticato dupliciter. Primo quia plus avide sumitur ex quadam degluciendi voracitate. Secundo quoniam deglutitus imperfecte digeritur, et per consequens corpus ad multas preparat passiones » (Regimen sanitatis ad regem Aragonum..., p. 430). 
seulement être attentif à l'heure du repas et à la quantité, mais aussi à la qualité des aliments, c'est-à-dire à consommer ce qui convient au corps selon l'exigence de sa nature et du moment, et à éviter ceux qui ne conviennent pas ${ }^{44}$. Il donne à ce propos l'exemple des nourritures piquantes et salées, que doit éviter celui qui souffre d'hémorroïdes. Cette attention à la nature propre de l'aliment et à sa faculté d'être assimilé conduit aussi les praticiens à réfléchir à la nature même de l'aliment, par lui-même facteur de risque.

\section{Pour une définition médicale de l'aliment et du médicament}

Aux yeux des médecins du Moyen Âge, mais pas sans doute à ceux de leurs contemporains, tout ce qu'on mange n'est pas propre à la consommation et des discordances peuvent se faire jour entre les recommandations des premiers et la réalité des habitudes alimentaires des seconds. Il y a là manifestement une perception du risque qui n'est pas partagée. C'est l'ambivalence même du cibus qui conduit les praticiens à proposer une sorte de hiérarchie qui repose sur les interactions réciproques, lors du processus de digestion, entre la substance ingérée et le corps. Chez certains auteurs, les distinctions, a priori classiques entre aliments et médicaments, deviennent assez subtiles, à l'image des classifications que propose le médecin Ugo Benzi (1376-1439) dans son commentaire au premier livre du Canon d'Avicenne ${ }^{45}$. Selon le maître originaire de Sienne, les substances que l'homme est susceptible d'ingérer sont de quatre types (fig. 2). Seules les deux dernières retiendront vraiment notre attention. En effet, il distingue d'abord les poisons et les médicaments vénéneux (medicina venenosa), qu'on ne saurait qualifier à proprement parler d'aliment, même s'il existe souvent, comme chez Ugo Benzi, des questions scolastiques du type « est-ce que le venin nourrit ? ». Ces substances naturellement nocives se caractérisent par le fait de ne pas être transformées par la chaleur corporelle lors du processus digestif et d'être en revanche capables d'affaiblir le corps en lui faisant subir une transformation mortelle (transmutatio mortifera) qui détruit sa vertu et occasionne la mort ${ }^{46}$. La deuxième catégorie décrite par Ugo Benzi regroupe les médicaments « absolus »(medicinae absolutae),

44. «Non solum autem hora refeccionis et quantitas sed eciam et qualitas ciborum debet attendi, sic scilicet ut illi sumantur qui corpori congruunt secundum exigenciam nature ipsius et temporis et qui non congruunt evitentur » (ibid., p. 431).

45. Et plus particulièrement au chapitre IX, fen II, doctrine II du premier livre, consacré à « ce qui provient de ce qui est mangé et bu » (De his que proveniunt ex his que comeduntur et bibuntur).

46. «[...] est venenum absolute quod mutat corpus et non mutatur a corpore ad sensum dictum, ita tamen quod ipsa tandem destruit corporis virtutem ita de reliquis exemplis propositis » (Ugo Benzi, In primam primi. Cum tabula, Florence, mandato et expensis nobilis viri d ${ }^{\text {ni }}$ Luceantonij de Giunta florentini, 1523, $\left.\mathrm{f}^{\circ} 74 \mathrm{r}^{\circ} \mathrm{a}\right)$. Le Canon constitue $1^{\prime} \mathrm{un}$ des textes majeurs de l'enseignement médical dans les universités médiévales. 


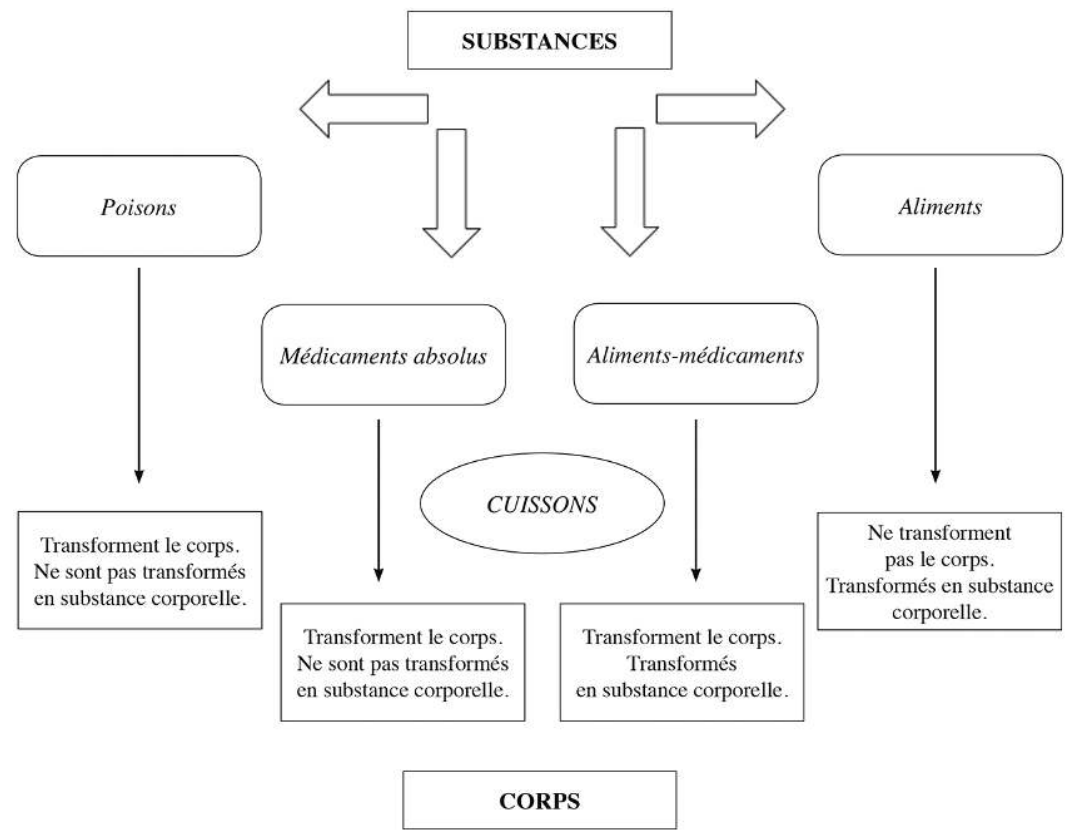

Fig. 2. Les catégories de substances ingérables d'après Ugo Benzi

c'est-à-dire des simples qui sont consommés à des fins thérapeutiques; du fait de leurs qualités premières, voire de particularités qui tiennent à ce que, depuis Avicenne, on qualifie de «forme spécifique ${ }^{47}$ », ils servent avant tout à corriger la complexion non tempérée du patient. Ils transforment le corps qui les reçoit mais ne sont pas altérés ou transformés par le processus digestif. En un mot, ils ne nourrissent pas.

Les deux dernières catégories regroupent des substances qu'on ingère plus ou moins couramment à des fins nutritives : d'abord les « alimentsmédicaments » (cibi medicinales) qui visent aussi à corriger le déséquilibre des complexions. Ils sont généralement affublés d'une complexion propre souvent située du côté de l'excès sur l'échelle des degrés des qualités premières ${ }^{48}$. Du fait de ces propriétés particulières, ils sont donc, à l'image

47. Cette notion, très utilisée dans le domaine de la pharmacopée, permet de réduire le champ d'action des qualités premières, en soutenant qu'un produit (aliment ou médicament, simple ou composé) n'est pas seulement défini par l'action de ses qualités premières, mais aussi par une propriété acquise après la détermination de la complexion et qui se définit par son effet. Sur cette notion, voir N. Weill-Parot, Les "Images astrologiques » au Moyen $\hat{A} g e$ et à la Renaissance. Spéculations intellectuelles et pratiques magiques (XII - XVe siècle), Paris, 2002, p. 450-455.

48. Chaque qualité est définie par une échelle de quatre degrés. Toutefois, d'un auteur à l'autre, les degrés, voire les qualités attribuées, peuvent varier pour un même aliment. 
des médicaments, considérés comme des correctifs et leur consommation est recommandée dans les cas de déséquilibre de la complexion, c'est-à-dire pour des malades qu'il convient, par une alimentation contraire, de ramener vers leur état naturel ou état de santé (forma sanabilis). Cet effet correctif s'explique aussi par la réaction qu'ils suscitent et qu'ils subissent lors du processus de digestion : à la différence des aliments qualifiés par Ugo Benzi de «naturels », entièrement assimilés par le corps sans que, pour autant, ils l'altèrent, les « aliments-médicaments », comme les médicaments, transforment le corps ; toutefois, ils subissent aussi une mutation qui les change en substance corporelle, à la différence des « médicaments absolus » (medicinae absolutae) ${ }^{49}$. Ces aliments-médicaments participent donc d'un double registre : comme les médicaments, ils agissent principalement pour corriger ; comme les aliments, ils ont une certaine valeur nutritive, même si elle est dans l'ensemble considérée comme médiocre.

Il en est ainsi des fruits en général à propos desquels le discours médical est plutôt négatif ${ }^{50}$ : on considère qu'ils sont souvent difficiles à digérer et provoquent des flatulences. Du fait de leur complexion et des effets qu'ils produisent sur le corps, il est recommandé de ne pas les consommer comme aliments, mais par «voie de médecine », de manière modérée et en considérant toujours l'utilité que l'on est en droit d'espérer de tels usages. Arnaud de Villeneuve déclare qu' « en effet, ils ne doivent pas être consommés pour la volupté, mais pour une utilité évidente, car l'usage des fruits pour la volupté empêche la conservation de la santé ${ }^{51}$. L' « utilité » dont il est ici question est uniquement pensée comme préventive, voire thérapeutique. Selon leurs propriétés intrinsèques, les fruits peuvent, à l'image des melons, être mangés pour apaiser le bouillonnement du sang (ad mitigandum fervorem sanguinis), pour briser l'acuité (frangendum acumen) de la colère s'ils sont acides, ou encore plus largement pour comprimer ou relâcher. Il n'est pas de variété de fruit citée par le maître montpelliérain qui n'ait quelque effet spécifique sur le corps. Au total, ces cibi medicinales

49. «Quod mutet et mutetur a corpore, [...] hoc est duobus modis : quia aut id convertitur tandem in similitudine corporis et est medicinale nutrimentum, aut non convertitur in similitudinem corporis et est medicina absolute... Quod mutat corpus et mutatur a corpore ita ut tandem non convertatur in substantiam corporis sed eius virtus et operatio destruantur sit medicina absolute » (Ugo Benzi, In primam primi..., $\left.\mathrm{f}^{\circ} 73 \mathrm{v}^{\circ} \mathrm{b}-74 \mathrm{r}^{\circ} \mathrm{a}\right)$.

50. $C f$. M. Nicoud, «I medici medievali e la frutta : un prodotto ambiguo », dans I. Naso éd., Le parole della frutta. Storia, saperi, immagini, Turin, 2011, p. 91-108.

51. «Usus fructuum temperato corpori non congruit via cibi, sed pocius medicine, videlicet ad preservandum ipsum ab aliquo accidente nocivo quod ex concurrentibus potest timeri ; et ideo moderate et ordinate convenit ut eis utatur, considerata semper utilitate que speratur adquiri per sumpcionem ipsorum. Non enim ad voluptatem sumendi sunt, sed ad evidentem utilitatem, nam uti fructibus ad voluptatem conservacionem impedit sanitatis » (Regimen sanitatis ad regem Aragonum..., p. 442). 
permettent au médecin de ramener le corps du patient vers sa disposition naturelle.

La dernière catégorie retenue par Ugo Benzi concerne les « aliments naturels » (cibi naturales) ou encore les «nourritures absolues ». Eux seuls se voient reconnus une importante valeur nutritive, qui justifie qu'ils puissent entièrement se transformer au cours des digestions en différentes parties du corps $^{52}$. Cette conception générale ne signifie cependant pas que tous les aliments naturels se situent sur une même échelle et qu'il n'existe point, dans le discours médical, une sorte de hiérarchie alimentaire, qui regroupe souvent partiellement des habitudes socialement définies ; ainsi les aliments les plus subtils, souvent les plus chers, sont plus recommandés et valorisés que des nourritures plus grossières, mieux adaptées aux populations laborieuses. La diète des médecins, qui s'adresse principalement à des élites, se calque souvent sur les hiérarchies alimentaires que révèlent les habitudes de consommation médiévales et les justifie ${ }^{53}$.

La classification qu'opère Ugo Benzi dans son commentaire au premier livre du Canon d'Avicenne ne reflète cependant qu'improprement les usages contemporains et pose le problème du rapport entre les théories et les conceptions médicales d'une part et, de l'autre, les habitudes réelles des consommateurs.

\section{Enjeux et responsabilités}

S'il réserve plutôt ses propos théoriques à des confrères et aux étudiants en médecine, le praticien médiéval n'en cherche manifestement pas moins à sensibiliser un certain public au risque alimentaire et, plus particulièrement, aux dangers spécifiques que peut représenter pour lui telle ou telle substance. En définissant clairement, dans des ouvrages de vulgarisation, les dangers qu'on court à privilégier telle nourriture, il s'efforce sans doute de rendre visible et compréhensible un risque dont la perception ne va pas de soi : pourquoi se méfier de ce qui a bon goût et de

52. «Dicit [Avicenna] primo quod illud quod primo a materia operatur est illud quod in corpore hominis convertitur a sui natura et recipit formam alicuius divisionis membrorum $\mathrm{i}[\mathrm{d}$ est $]$ alicuius membri contenti sub generali divisione membrorum. Ubi notandum quod cibus plerumque habet proprietatem currentem inter ipsum et cibatum propter quam est aptus pati a cibato talem passionem per quam convertitur in substantiam nutriti, ut patet ex Averroy 7 colliget, cap. 9 ubi dixit : "Et cibi naturales non conveniunt nobiscum nisi in tota substantia." Carnes ergo pullorum habent proprietatem per quam sunt apte pati a calore stomachi passionem qua convertuntur in verum chilum. [...] Illud quod patitur a corpore et non mutat corpus mutatione, de qua sit curandum, et convertitur in substantiam corporis est nutrimentum absolute $»\left(\mathrm{Ugo}\right.$ Benzi, In primam primi..., $\left.\mathrm{f}^{\circ} 68 \mathrm{r}^{\circ} \mathrm{b}, 74 \mathrm{r}^{\circ} \mathrm{a}\right)$.

53. Cf. B. Laurioux, Manger au Moyen Âge : pratiques et discours alimentaires en Europe aux XIVe et XV siècles, Paris, 2002. 
ce qui est beau d'aspect, bref pourquoi douter des merveilles de la nature ? Dans le même temps, il cherche aussi à responsabiliser le patient vis-à-vis de ses choix alimentaires.

\section{Les dangers de l'alimentation}

En divulguant auprès d'un public de non-spécialistes les rudiments d'un savoir sur l'alimentation, les médecins médiévaux répondent souvent à des sollicitations particulières, lorsque la rédaction d'un régime est le résultat d'une demande spécifique. Plus largement, ces ouvrages correspondent aussi à un besoin d'assurance, à une envie de connaissance, à un souci de prévention de la santé que l'on voit poindre et s'affirmer chez les élites aux xive et $\mathrm{xv}^{\mathrm{e}}$ siècles. La vertu des aliments, les plus communs mais aussi les plus rares, les meilleures variétés à consommer, ou encore les manières de les préparer, y voisinent avec la description des effets néfastes que ces mêmes nourritures peuvent produire sur l'organisme.

Au premier rang de ces dangers spécifiques, se situe l'aliment corrompu, plus facile à identifier, celui que traquent aussi les autorités urbaines. L'odeur, le goût ou encore la vue, de même que la provenance, constituent ici des instruments déclarés valides d'appréciation de la qualité d'un produit, et le recours aux sens est fortement recommandé au consommateur ${ }^{54}$. Ainsi l'apparence de l'aliment, lorsqu'il se marque ou se putréfie, ou encore la présence de vers, signalent sa dangerosité ${ }^{55}$. Les médecins précisent aussi parfois la durée au-delà de laquelle une nourriture conservée ne peut plus être consommée : il s'agit de sortes de « date de péremption » avant la lettre, qui indiquent par exemple qu'il ne faut pas consommer de farine et de fleur de farine, de son ou encore de pâtes de plus de six mois, d'amidon de plus de douze mois ou encore de riz de plus de huit à dix mois selon qu'il est ou non complet ${ }^{56}$.

Pourtant les dangers ne sont pas toujours aussi faciles à percevoir et la nocivité dépasse souvent la simple notion de corruption. Il est des

54. À l'image de la consommation de poissons dont il faut éviter en général les espèces lacustres, de rivière à faible débit, proches des lieux habités.

55. "Secunda vero regula est quod abstinendum est a fructibus quandocumque multiplicantur in eis vermes aut interius tabefiunt quod in vulgari catalanorum dicitur entecar; et cognoscitur per hoc quia fructus interius non est naturaliter coloratus nec habet naturam medullam » (Regimen sanitatis ad regem Aragonum..., p. 443).

56. «In predictis [granis panificis] eciam omnibus est cavendum ne vetustate corrupta sint vel appropinquent corrupcioni. Et ideo farina et furfur et simila et tri que plus sex mensibus fuerint reservata, vitari debent a corporibus temperatis atque colericis ; et amidum si plus quam duodecim menses habuerit; et rizi si plus quam decem et octo mensibus fuerit extra corticem reservatum, et maxime in regionibus calidis. Generaliter vero tenendum est quod, si predicta omnia fumosi odoris percipiantur esse vel acuti saporis aut terrei, semper sunt vitanda » (ibid., p. 438). 
nourritures qui ne conviennent pas à certains organismes et très tôt, tout particulièrement à partir du xiv ${ }^{\mathrm{e}}$ siècle, les médecins ont commencé à user de préparations spécifiques comme d'un moyen de corriger et rendre consommables ces aliments. Cette « cuisine médicale », comme la qualifie Bruno Laurioux ${ }^{57}$, « est à même de prolonger, de corriger ou d'accélérer le processus de digestion, conçu lui-même comme une coction ${ }^{58}$ ». D'un auteur à l'autre toutefois, l'importance accordée à ces aspects peut varier d'autant que certains aliments paraissent en quelque sorte « irrécupérables » et sont prohibés de l'alimentation des hommes à complexion tempérée ${ }^{59}$. Nul doute cependant que ceux qui ont frayé avec des milieux de cour, à l'image d'Arnaud de Villeneuve auprès du roi Jacques II d'Aragon ou du padouan Michel Savonarole (1385-1468) auprès du duc Borso d'Este à Ferrare, sont plus sensibles et réceptifs aux apports culinaires : leurs régimes attribuent en effet une grande place à ces préparations, sans pour autant nier aussi les dangers nouveaux que peut représenter pour le corps l'émergence de cet art culinaire ${ }^{60}$, susceptible de modifier les goûts et les appétences des consommateurs.

Plus largement, ces régimes de la fin du Moyen Âge ont tendance à s'adapter plus étroitement aux usages alimentaires contemporains, à faire plus de place aux nourritures communes des tables des riches, à ne plus guère parler en revanche des aliments peu courants. Sous peine aussi de ne pas être lus ou de paraître obsolètes, les médecins sont également obligés de statuer sur de nouvelles consommations (à l'image des propos tenus sur les pâtes ${ }^{61}$ ) ou sur de nouvelles variétés, comme les agrumes, de plus en plus

57. B. Laurioux, «La cuisine des médecins du Moyen Âge », dans Maladies, Médecines et Sociétés. Approches historiques pour le Présent. Actes du VI Colloque d'Histoire au Présent, Paris, 1993, t. II, p. 136-148.

58. Id., "Cuisine et médecine au Moyen Âge : alliées ou ennemies? » Cahiers de recherches médiévales, 13 (2006), p. 223-238. Il s'agit aussi de dépasser les idées reçues selon lesquelles la consommation d'aliments avariés ou faisandés était importante et leur goût maquillé par des sauces et des épices.

59. "Anates vero et fulce magis autompno conveniunt, sed corporibus temperatis nunquam expedit anatibus uti vel fulcis» (Regimen sanitatis ad regem Aragonum..., p. 454).

60. Cf. B. Laurioux, Le Règne de Taillevent : livres et pratiques culinaires à la fin du Moyen Âge, Paris, 1997 ; Id., Les Livres de cuisine médiévaux, Turnhout, 1997 (Typologie des Sources du Moyen Âge occidental, 77).

61. O. Redon, B. Laurioux, "L'apparition et la diffusion des pâtes sèches en Italie (xiiie- xiv $v^{\mathrm{e}}$ siècles) », dans D. Meeks, D. Garcia éd., Techniques et économie antiques et médiévales : le temps de l'innovation, Paris, 1996, p. 101-108; Eid., « La constitution d'une nouvelle catégorie culinaire ? Les pâtes dans les livres de cuisine italiens de la fin du Moyen Âge », Médiévales, 16-17 (1989), p. 51-75 ; S. Serventi, F. Sabban, Les Pâtes. Histoire d'une culture universelle, trad. fr., Arles, 2001. 
utilisés en cuisine et dont, de ce fait, « il faut dire quelque chose », comme le reconnaît Michel Savonarole ${ }^{62}$.

Nouvelles catégories alimentaires et inventions culinaires donnent ainsi naissance à de nouveaux dangers, que le recours aux conseils des médecins, perceptible à travers la rédaction de régime ou par le biais de la présence réelle de praticiens auprès des princes et jusque dans leurs cuisines, est censé écarter. Pourtant, malgré sa diffusion, la littérature diététique pose la question de sa propre application. Ici se mesurent les limites du pouvoir du médecin lorsque, en matière de santé et d'alimentation, il ne peut pas agir sans faire cas des propres choix du patient.

\section{Risque et responsabilités}

Textes médicaux et documentation de la pratique témoignent de l'effort des praticiens pour tenter de responsabiliser le patient en matière de santé personnelle et, notamment, de choix alimentaires. Certes, du côté des sources pratiques, les occurrences ne sont pas très nombreuses, du fait tout simplement de la difficulté à repérer ces types de mentions, par exemple dans les archives notariales qui conservent des contrats passés entre patients et médecins ${ }^{63}$. Toutefois, les rares informations trouvées témoignent du fait qu'au moment de l'établissement du contrat, la question du « risque alimentaire » a pu être envisagée quand elle pouvait remettre en question la thérapie proposée. Les deux cas que j'évoquerai rapidement, issus de l'Archivio di Stato de Palerme, concernent des actes signés dans la première moitié du $\mathrm{Xv}^{\mathrm{e}}$ siècle ${ }^{64}$.

Dans le premier, l'énumération des engagements réciproques des deux contractants est suivie d'une clause supplémentaire qui stipule que le patient, en l'occurrence lui-même notaire, s'engage à ne pas consommer certains aliments (res comestibiles) déclarés par le praticien « inutiles », et dont ce dernier doit fournir la liste ${ }^{65}$. Si le malade contrevenait à son engagement, il n'est pas pour autant prévu que cela puisse être considéré comme une clause libératoire, qui ferait alors peser sur le patient l'entière responsabilité d'un possible échec de la cure.

62. «Citrum \& si non sit apud omnes in usu ; tamen apud nobiles hodierno tempore cum cibariis, \& assatis comeditur ; quare de eo aliquid dicendum est » (Michel Savonarole, Practica maior, Venise, apud Vincentium Valgrisium, $\left.1560, \mathrm{f}^{\circ} 21 \mathrm{v}^{\circ} \mathrm{b}\right)$.

63. Sur ce type de document, voir G. Pomata, La Promessa di guarigione. Malati e curatori in antico regime. Bologna, XVI-XVIII, Bari, 1994.

64. Documents cités par H. Bresc, « Documents siciliens », Razo, 4 (1984), Le Corps souffrant : maladies et médications, p. $109, \mathrm{n}^{\circ} 2$, et p. $111, \mathrm{n}^{\circ} 17$.

65. «Pactum similiter quod idem notarius Thomasius debeat se astinere a rebus comestibilibus sed inutilibus sibi declarandis per dictum magistrum Fridericum infra dictum tempus »(ASP, Notai Defunti b. 31 ; acte signé à Corleone, devant le notaire Giacomo de Pittacolis, le 23 novembre 1405). 
La deuxième occurrence est plus intéressante encore puisque sont énumérés dans le contrat même un certain nombre d'aliments utiles ou au contraire à interdire : de manière positive, le vin à consommer tempéré, c'est-à-dire avec de l'eau ; de manière négative, les poissons et le thon salés qui doivent être au contraire prohibés de l'alimentation d'un malade atteint d'une plaie à la jambe ${ }^{66}$. Surtout, l'acte cette fois responsabilise le patient et le rend coupable d'un éventuel échec : contrevenant à son engagement en matière diététique (faciendo disordines), ce dernier, même s'il n'est pas guéri, devra verser au chirurgien les deux sous dont il devait s'acquitter en cas de guérison.

Plus fréquentes encore se révèlent les mises en garde dans les milieux privilégiés, où l'on a accès de manière quotidienne au praticien. Il n'est pas étonnant donc que ce soit dans les espaces curiaux, où sont appointés des médecins et où sont rédigés, adressés et diffusés les régimes de santé, que se trouvent des traces de ces mesures de précaution en matière alimentaire ${ }^{67}$. Divers passages empruntés à l'ordonnance de l'hôtel de l'héritier ducal de Milan, Galeazzo Maria Sforza, rédigée à la fin des années 1440, font ainsi état de ce type de mesures. À plusieurs reprises, des conseils ou des recommandations sont proposés à propos de l'alimentation qui font intervenir aussi bien la vigilance des gouverneurs et des pédagogues, que celle bien sûr des médecins constamment présents auprès de l'enfant et notamment au moment des repas ${ }^{68}$. Ainsi, dans le texte programmatique rédigé par un familier, Franchino Caimi, il est question des dangers d'une alimentation trop riche et diversifiée pour un jeune enfant (âgé de moins de cinq ans à l'époque) ${ }^{69}$; il est aussi fait mention des périls que peuvent constituer des animaux morts dont on ne connaît pas la provenance : sans information sur le sujet, il faut se garder d'en autoriser la consommation ${ }^{70}$.

66. ASP, Notai spezzone 277 (Palerme, 15 avril 1447).

67. Pour une étude de la présence médicale à la cour des ducs lombards, $c f$. M. Nicoud, Le Prince et les médecins. Pensée et pratiques médicales à Milan (1402-1476), Rome, 2013 (CEFR, 488).

68. «Al fatto del vivere suo, quantunque sia importantissimo, non ne pare pero necessario de fare gran mentione, considerandose de la diligentia vostra et vigilantia di quelli nostri phisici quali stano de continuo apresso de la persona del nostro fiolo, ho la piu parte de loro et maximamente ale hore del mangiare et quando se parte de casa... » (éd. M. Ferrari, «Per non manchare in tuto del debito mio ». L'educazione dei bambini Sforza nel Quattrocento, Milan, 2000, p. 64).

69. «Ve ricordamo pero, acio ne habiate casone de conferire insieme et servare quella regula et ordine vi payra tuti vuy, che la diversitate di cibi ali puti di questa eta e molto nociva, pur el lasiamo et ala discretione vostra et de loro, havendo respecto ala eta e conditione desso Signore et farite che li seschalchi in ogni deliberatione in fra vuy facta, per rispecto di cibi pertinenti ala cusina, si trovino de presente acio non possino diviarse da li ordini haverite fra vuy facti » (ibid.).

70. «Item ve recordamo che accadendoli li sia presentato cossa alcuna morta mangiativa vogliati considerare el locho et da chi sia presentato prima se conceda ne per vuy ne per li 
Dans l'amendement à ces mesures proposées par Messer Pallavicino, lui aussi gouverneur de l'héritier, il est écrit qu'il faut seulement ajouter « que pour tout ce qui se passe en cuisine, cela se fasse avec la participation des médecins pour ce qui regarde la santé de Galeazzo ${ }^{71}$. L'ordonnance est enfin également corrigée par un médecin ducal, Cristoforo da Soncino, attaché à la personne de Galeazzo. Parmi ses observations, on note une réflexion à propos des animaux morts, pour signaler que ce ne sont pas les seuls à pouvoir « se corrompre et contaminer ${ }^{72}$ », et qu'il faut par conséquent être attentif plus largement à la qualité et à la fraîcheur de l'ensemble des nourritures, aussi bien carnées que végétales. Les recommandations imposées dans cette cour italienne se retrouvent également ailleurs, comme en témoigne une ordonnance pour l'hôtel du roi d'Angleterre Édouard IV (1461-1483), qui prévoyait que le praticien réponde aux questions sur la nature et l'effet des nourritures, et parle également avec le maître queux pour choisir les mets les plus appropriés au souverain ${ }^{73}$.

Pourtant, en dépit des mises en garde répétées et des propositions de réglementation des pratiques alimentaires, le respect des recommandations diététiques des praticiens, leurs conseils et autres régimes ne sont pas toujours suivis d'effets, surtout lorsqu'ils s'adressent à des patients en parfaite santé. Entre les goûts des individus et les recommandations des médecins, entre les plaisirs de la bouche et les dangers de la table, il y a d'inévitables conflits d'intérêts où la perception du risque alimentaire, plus que la notion elle-même, n'est pas une donnée univoque ni facile à appréhender ; sa définition ne va pas de soi, selon que l'on est médecin ou patient, mais aussi selon que ce dernier est malade ou en bonne santé. Aussi les interdictions auxquelles le risque peut donner lieu ne sont-elles pas a priori acceptées sans discussion et le régime alimentaire fait souvent l'objet d'une négociation entre celui qui tente de l'imposer et celui qui doit le suivre ${ }^{74}$.

Pour un malade, il n'est pas de chemin plus direct vers la santé que de se passer de médecin [...]. Un tel dit qu'il faut s'abstenir de fruits, tel autre de

seschalchi chel Signore ne mangi [...] acio stiano con li ogji aperti ala salute del preffato nostro fiolo » (ibid.).

71. «Lo 13 capitulo dico stare bene, ma dovergli fare gionto : ordinare a la cusina quanto a le imbandisone che tuto se facia con participatione de li medici per quanto specta a la sanita soa » (ibid., p. 66).

72. «Et Decimo septimo fa mentione del mangiare cose presentate morte quasi se ponga per fermo le vive non potersi vitiare et contaminare, alche é falsissimo, verum non ita faciliter» (ibid., p. 69).

73. Cité par B. Laurioux, « Cuisine et médecine... », p. 226.

74. Pour une analyse de cas à Milan, à propos de l'éducation de Galeazzo Maria Sforza, voir M. Nicoud, « Les savoirs diététiques à la cour... ». 
légumes et d'herbes potagères, sans lesquels, aux yeux d'un grand nombre de gens et surtout de nos compatriotes, la nourriture la plus raffinée et la plus sophistiquée perd tout attrait. [...] Or voilà qu'un de nos oracles a fait tout son possible pour les rendre infâmes et suspectes aux yeux de l'ensemble des mortels, sous prétexte qu'elles ne lui plaisent pas ou ne lui étaient d'aucun profit. Nos princes n'osent ni roter, ni cracher sans la permission des médecins, et pourtant ils ne vivent ni mieux ni plus longtemps que leurs prédécesseurs. Les médecins, de leur côté, président les tables des rois, en vertu de l'autorité que l'usage leur a conférée : ils ordonnent, interdisent, menacent, tonitruent, argumentent, s'indignent et imposent à leurs maîtres des règles qu'ils sont les premiers à transgresser - règles dont l'observance, comme nous le voyons, abrège la vie des rois et prolonge leurs maladies ${ }^{75}$.

Ces invectives de Pétrarque, raillant l'incompétence et l'absence d'uniformité dans les propos des médecins, ne fournissent qu'un témoignage de plus, enlevé certes, sur l'incompréhension, voire sur le refus d'obéissance, que suscitent les mises en garde des praticiens. Que ce soit à travers leurs discours, leurs écrits ou dans le cadre de leur pratique, les médecins ont toujours accordé une grande place à l'alimentation, à ses vertus comme à ses dangers, soulignant sa nature ambiguë et l'usage déraisonné que l'homme pouvait en faire. Si la prise en compte du risque est au cœur de ses conseils et de son action, s'il n'ignore pas que son art, plus que tout autre, est périlleux pour la santé, le praticien sait aussi devoir compter sur l'entière obéissance de son patient pour espérer la réussite de sa cure. Dans un domaine alimentaire peut-être plus que d'autres soumis à l'innovation et à la diversité - qu'il s'agisse de l'attrait de nouvelles variétés, des délices d'une cuisine épicée ${ }^{76}$, ou bien encore de la quantité de plats servis au cours d'un même banquet ${ }^{77}$-, la participation active du consommateur s'avère encore plus indispensable et les risques encourus certainement nombreux. Ces spécificités expliquent sans doute aussi qu'au-delà d'un propos générique qui, d'un régime à l'autre ou d'un conseil à l'autre, peut apparaître aux yeux d'un lecteur contemporain pour le moins répétitif, le propos médical sur l'alimentation tende au contraire à s'adapter aux habitudes de consommation de ses contemporains afin de concilier, autant que possible, les goûts des uns et les connaissances des autres.

Marilyn Nicoud - Université d'Avignon et des Pays du Vaucluse, UMR 5648

75. Lettre adressée à Boccace en 1365 (Pétrarque, Lettres de la vieillesse, IV-VII, II, Paris, 2003, V, 3).

76. Cf. B. Laurioux, Manger au Moyen Âge...

77. Voir par exemple les diverses contributions publiées dans R. Comba, A. M. Nada Patrone, I. Naso éd., La mensa del Principe. Cucina e regimi alimentari nelle corti sabaude (xiii-xv secolo), Cuneo, 1996. 


\section{L'alimentation, un risque pour la santé ? Discours médical et pratiques alimentaires au Moyen Âge}

Pourquoi s'interroger sur l'existence d'une notion de risque alimentaire dans le discours des médecins du Moyen Âge tardif ? Si la documentation archivistique communale ne fournit pas de trace véritable d'expertise médicale en matière de sécurité alimentaire, alors que les autorités publiques ont tenté de mettre en place des mesures de précaution et de contrôle, les ouvrages médicaux et principalement diététiques fournissent cependant le témoignage d'une réflexion sur les dangers causés par l'alimentation à une échelle individuelle. À travers des traités aussi bien savants que de vulgarisation, les médecins distinguent les aliments des médicaments et des aliments-médicaments et s'efforcent de sensibiliser leurs patients à la nécessité de réguler leurs consommations et de connaître les qualités des aliments : élément essentiel à la vie, utilisée aussi bien pour conserver la santé que dans un cadre thérapeutique, l'alimentation est en effet aussi potentiellement un facteur de risque sanitaire qu'il convient de circonscrire.

Alimentation - corps - diététique - médecine - risques - santé

\section{Food, a Risk for Health ? Medical Discourse and Dietary Practices in the Middle Ages}

Why question the existence of a concept of food risk in the discourse of the physicians of the late Middle Ages ? If the communal archives provide no trace of medical expertise regarding food safety, while public authorities have tried to set up precautionary measures to control public health, however medical and mainly dietary books supply the testimony of a reflection on the dangers caused by food at an individual level. Through learned works as well as didactic texts, authors distinguish medicine from food and foodmedicine, and try to educate their patients on the necessity to regulate their consumption and be aware of the qualities of food : food, which is essential to life, is both used in medieval medicine for maintaining health and in a therapeutic way. But it is also, potentially, a sanitary risk factor which has to be defined.

Body - dietetics - food - health - medicine - risk 